\title{
Bayesian least squares deconvolution
}

\author{
A. Asensio Ramos ${ }^{1,2}$ and P. Petit ${ }^{3,4}$ \\ 1 Instituto de Astrofísica de Canarias, 38205 La Laguna, Tenerife, Spain \\ e-mail: aasensio@iac.es \\ 2 Departamento de Astrofísica, Universidad de La Laguna, 38205 La Laguna, Tenerife, Spain \\ ${ }^{3}$ Université de Toulouse, UPS-OMP, Institut de Recherche en Astrophysique et Planétologie, 31400 Toulouse, France \\ ${ }^{4}$ CNRS, Institut de Recherche en Astrophysique et Planétologie, 14 avenue Édouard Belin, 31400 Toulouse, France
}

Received 24 April 2015 / Accepted 12 September 2015

\section{ABSTRACT}

\begin{abstract}
Aims. We develop a fully Bayesian least squares deconvolution (LSD) that can be applied to the reliable detection of magnetic signals in noise-limited stellar spectropolarimetric observations using multiline techniques.

Methods. We consider LSD under the Bayesian framework and we introduce a flexible Gaussian process (GP) prior for the LSD profile. This prior allows the result to automatically adapt to the presence of signal. We exploit several linear algebra identities to accelerate the calculations. The final algorithm can deal with thousands of spectral lines in a few seconds.

Results. We demonstrate the reliability of the method with synthetic experiments and we apply it to real spectropolarimetric observations of magnetic stars. We are able to recover the magnetic signals using a small number of spectral lines, together with the uncertainty at each velocity bin. This allows the user to consider if the detected signal is reliable. The code to compute the Bayesian LSD profile is freely available.
\end{abstract}

Key words. stars: magnetic field - stars: atmospheres - line: profiles - methods: data analysis

\section{Introduction}

The phenomena taking place in the atmospheres of solar-type stars is controlled by magnetic fields. One of the most straightforward ways of detecting and measuring such magnetic fields is through spectropolarimetry. The observation of the intensity (Stokes $I$ ), linear polarization (Stokes $Q$ and $U$ ), and circular polarization (Stokes $V$ ) allows us to remotely infer the strength and orientation of magnetic fields.

During the last decades, we have been able to detect magnetic fields in cool stars. The first efforts (Donati et al. 1992) detected the presence of polarimetric signals in the most active of them. In these cases, it was necessary to reach a very high polarimetric sensitivity to detect these signals in individual spectral lines. In general, however, the polarization level in almost all cool stars is below our detection limit and we require postprocessing techniques to enhance the detection rate. The first solution to this problem was the development of multiline techniques (Semel 1989; Semel \& Li 1996), which averaged many photospheric spectral lines to improve the effective polarimetric sensitivity. This technique works under the assumption that noise is uncorrelated and adds up to zero when many spectral lines are summed. Meanwhile, the spectral line itself presents correlations that will make it appear above the noise after adding many spectral lines. This idea was later improved with the introduction of the least squares deconvolution technique (LSD; Donati et al. 1997), which has facilitated the detection of polarimetric signals in a wide variety of stars and the generation of a set of interesting surveys for the detection of magnetic fields in different stellar types (e.g., Wade et al. 2012; Marsden et al. 2014; Martins et al. 2015).

Recently, the LSD method has been critically analyzed by Kochukhov et al. (2010), who pointed out the difficulties in the interpretation of the final LSD profile in terms of a normal spectral line. In recent years, several variations of the LSD method have been presented. These methods try to overcome some of the fundamental problems with LSD. For instance, Sennhauser et al. (2009) suggested introducing an improvement to deal nonlinearly with the presence of blends in spectral lines, much closer to what really happens during the formation of the spectral line. Carroll et al. (2008) and Martínez González et al. (2008) generalized LSD to account for the deviations from the assumption of a single common profile for all spectral lines. This was done by using the principal component analysis (PCA) decomposition of all the considered lines and truncating the decomposition to get rid of the noise. Semel et al. (2009) introduced the idea of multiline Zeeman signatures as detectors of the presence of magnetic fields. Finally, Kochukhov et al. (2010) introduced multiprofile LSD, a generalization of LSD that models each spectral line as the linear addition of several profiles, very similar to the PCA approach. These authors also discuss a simple modification of LSD to regularize the solution using a first-order Tikhonov regularization, which favors smooth solutions.

In this paper we treat the LSD from a Bayesian perspective and consider the use of a flexible prior for the expected LSD profile. This introduces a regularization that automatically adapts to the data. The method is fast and competitive in computing time with LSD. Given that the method we propose is fully Bayesian, one of the main advantages is that we are able to give sensible error bars to the detection of LSD signatures.

\section{Bayesian LSD}

The LSD technique is based on assuming that the circular polarization profiles of a set of selected spectral lines from a star correspond to a common basic Zeeman signature. The spectral 
shape of this signature does not change and only the amplitude is modified from line to line. The validity of this approximation is based on two assumptions. First, the line has to be in the so-called weak field regime, which happens when the magnetic field is sufficiently weak so that the Zeeman splitting is smaller than the Doppler width of the spectral line. Second, the line has to be weak so that no saturation effects are detected. Under these simplifying assumptions, the radiative transfer equation can be solved analytically and it can be demonstrated that the Stokes $V$ profile is proportional to the wavelength derivative of the intensity profile (e.g., Landi Degl'Innocenti \& Landolfi 2004). In this case Donati et al. (1997) demonstrated that it is possible to write the Stokes $V$ profile for line $i$ as

$V_{i}(v)=\alpha_{i} Z(v)$,

where $v$ is the displacement with respect to the central wavelength of the line in velocity units and $\alpha_{i}$ is a proportionality constant that depends on the properties of the line. Donati et al. (1997) also showed that these constants are $\alpha_{i}=\lambda_{i} d_{i} g_{i}$, with $\lambda_{i}$ the central wavelength of the line, $d_{i}$ the depth of the line, and $g_{i}$ the effective Landé factor of the line.

With a model for all the lines at hand, we can write the generative model. It states how a given observed spectral line is modeled in terms of some parameters. We assume that we observe $N_{\text {lines }}$ spectral lines, each one sampled at $N_{v}$ velocity positions. Then, we model our measurements with

$V_{i}\left(v_{j}\right)=\alpha_{i} Z\left(v_{j}\right)+\epsilon_{i j}$,

which indicates that our measured Stokes $V$ profile at a given velocity position and for a certain spectral line is just a weight times the common signature at this very same velocity with some added noise, $\epsilon_{i j}$. This noise can potentially be different for all velocity positions of all the spectral lines and can even accommodate the more general case in which there is nonzero correlation between different pixels. We note that the generative model can be written in matrix notation as

$\boldsymbol{V}=\mathbf{W} \boldsymbol{Z}+\boldsymbol{\epsilon}$

where the matrix $\mathbf{W}$ is given by

$\mathbf{W}=\left[\begin{array}{ccccc}\alpha_{1} & 0 & 0 & 0 & \ldots \\ \alpha_{2} & 0 & 0 & 0 & \ldots \\ \alpha_{3} & 0 & 0 & 0 & \ldots \\ \cdots & \ldots & \ldots & \ldots & \ldots \\ 0 & \alpha_{1} & 0 & 0 & \ldots \\ 0 & \alpha_{2} & 0 & 0 & \ldots \\ 0 & \alpha_{3} & 0 & 0 & \ldots \\ \ldots & \ldots & \ldots & \ldots & \ldots\end{array}\right]$

In other words, the matrix elements are given by $W_{i j}=$ $\alpha_{j} \delta_{j, i \bmod j}$.

\subsection{Standard LSD}

It is very simple to obtain the standard LSD method in a Bayesian framework. To this end, we let the observations $V$ represent the Stokes $V$ profiles of line $i$ at wavelength $j$ ordered as $\boldsymbol{V}=\left[V_{1}\left(v_{1}\right), V_{2}\left(v_{1}\right), V_{3}\left(v_{1}\right), \ldots, V_{1}\left(v_{2}\right), V_{2}\left(v_{2}\right), \ldots\right]^{T}$. Likewise, we define the $\boldsymbol{Z}$ vector as the sampled common signature, given by $\boldsymbol{Z}=\left[Z\left(v_{1}\right), Z\left(v_{2}\right), \ldots\right]^{T}$. With these definitions, we can apply the Bayes theorem to obtain the probability distribution for the common signature given all the observed data

$p(\boldsymbol{Z} \mid \boldsymbol{V})=\frac{p(\boldsymbol{V} \mid \boldsymbol{Z}) p(\boldsymbol{Z})}{p(\boldsymbol{V})}$, where $p(\boldsymbol{Z} \mid \boldsymbol{V})$ is the posterior distribution for the common signature given the observations, $p(\boldsymbol{V} \mid \boldsymbol{Z})$ is the likelihood or sampling distribution (that gives the probability of getting a set of observed spectral lines conditioned on a common signature), $p(\boldsymbol{Z})$ is the prior distribution for the common signature (that encodes all the a priori information we have for $Z(v)$ ). Finally, $p(\boldsymbol{V})$ is the marginal likelihood or evidence that normalizes the posterior to make it a proper probability distribution. Given that the marginal likelihood does not depend on $\boldsymbol{Z}$, it is usual to drop it.

Even though the equations are valid for the general correlated case, we assume for simplicity that the noise is uncorrelated and with variance $\sigma_{i j}^{2}$ for line $i$ at velocity point $j$. Under this assumption, the likelihood can be written as

$p(\boldsymbol{V} \mid \boldsymbol{Z})=\mathcal{N}\left(\boldsymbol{V} \mid \mathbf{W} \boldsymbol{Z}, \operatorname{diag}\left(\boldsymbol{\sigma}^{2}\right)\right)$,

where $\mathcal{N}(\boldsymbol{x} \mid \boldsymbol{\mu}, \mathrm{C})$ represents a multivariate Gaussian distribution for variable $\boldsymbol{x}$ with mean vector $\boldsymbol{\mu}$ and covariance matrix $\mathbf{C}$. The vector $\sigma^{2}$ contains all the variances ordered exactly as in the case of $\boldsymbol{V}$. We note that if all pixels share the same noise variance, equal to $\sigma_{n}^{2}$, the likelihood simplifies to

$p(\boldsymbol{V} \mid \boldsymbol{Z})=\mathcal{N}\left(\boldsymbol{V} \mid \mathbf{W} \boldsymbol{Z}, \sigma_{n}^{2} \boldsymbol{I}\right)$.

If we assume that all $Z(v)$ profiles are equally probable a priori, the posterior probability for $Z(v)$ is

$p(\boldsymbol{Z} \mid \boldsymbol{V}) \propto \mathcal{N}\left(\boldsymbol{V} \mid \mathbf{W} \boldsymbol{Z}, \operatorname{diag}\left(\boldsymbol{\sigma}^{2}\right)\right)$,

where the posterior distribution is now seen as a distribution with respect to $\boldsymbol{Z}$. Equation (8) represents the full probabilistic LSD solution. Given the special structure of the matrix $\mathbf{W}$, it is very simple to compute the maximum a posteriori point estimate (the one that maximizes the posterior distribution) and one ends up with the known LSD result of Donati et al. (1997; see also Semel et al. 2009):

$Z_{\mathrm{LSD}}\left(v_{j}\right)=\frac{\sum_{i} \sigma_{i j}^{-2} \alpha_{i} V_{i}\left(v_{j}\right)}{\sum_{i} \sigma_{i j}^{-2} \alpha_{i}^{2}}$.

\subsection{Bayesian hierarchical model}

From the Bayesian point of view, the classical LSD method is just the maximum a posteriori solution to the inference problem when flat priors are used for the common signature $Z(v)$. In other words, the value of $Z(v)$ at one velocity position does not depend at all on the value at different velocity positions. This assumption does not capture some knowledge that we have from the physics of the problem. We know that there must be some correlation between different velocity positions because the Stokes $V$ profile are a consequence of the combined solution of the radiative transfer equation and the statistical equilibrium equations on the stellar atmosphere. The emission and absorption properties of the atmosphere inherently introduce correlation between different velocity bins. One consequence of this is the typical smooth shape of the $Z(v)$ profile, that we should somehow force in our solution.

In the Bayesian framework, we impose this condition by introducing a suitable prior distribution. A very convenient way to do it is by using a Gaussian process (GP; Rasmussen \& Williams 2005). Generally, a GP is a distribution over the subspace of functions that fulfill certain conditions. From the technical point of view, once a function is sampled at a finite number of points, a GP for the function $Z(v)$ is defined by the following multivariate normal distribution:

$p\left(\boldsymbol{Z} \mid \boldsymbol{\alpha}_{Z}\right)=\mathcal{N}\left(\boldsymbol{Z} \mid \boldsymbol{\mu}, \mathbf{K}\left(\boldsymbol{\alpha}_{Z}\right)\right)$, 


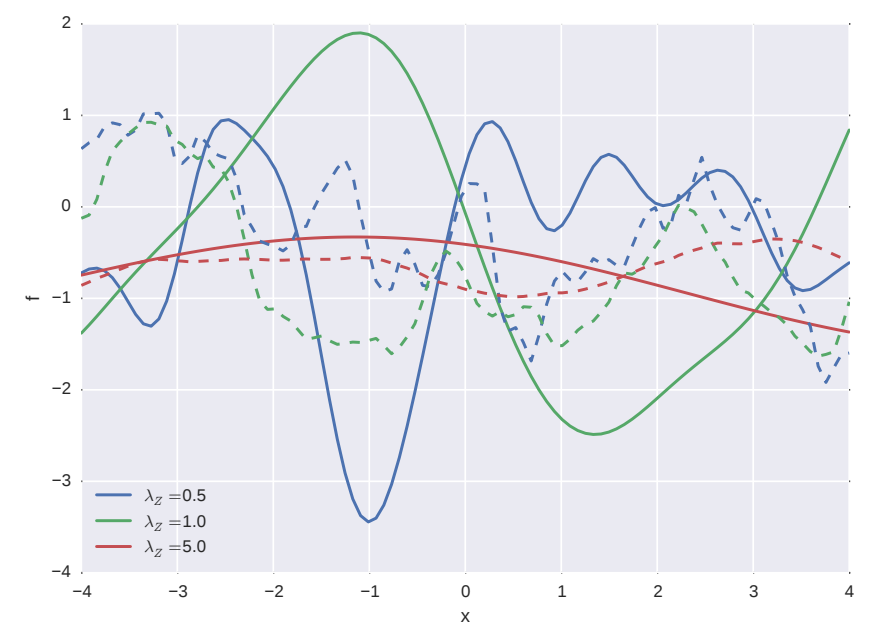

Fig. 1. Examples of functions extracted from GP priors with a squared exponential (solid lines) and Matérn with $v=3 / 2$ covariance functions. In both cases, $\sigma_{Z}=1$ and different values of $\lambda_{Z}$ are used. We note that $\lambda_{Z}$ acts as a scale over which the function varies. The functions extracted from the Matérn covariance function have a rougher behavior than those of the squared exponential one.

where $\boldsymbol{\mu}$ is the mean of the distribution, which is usually set to zero, and $\mathbf{K}\left(\boldsymbol{\alpha}_{Z}\right)$ is the covariance matrix, that depends on a set of parameters $\boldsymbol{\alpha}_{Z}$. The GPs are then defined by the specific covariance matrix. Instead of writing the full matrix, it is customary to define a function (the covariance function) that gives the value of the matrix elements. This covariance function has to fulfill certain conditions so that the ensuing covariance matrix is proper (for instance, the covariance matrix has to be symmetric). Widespread covariance functions are the squared exponential,

$K_{i j}\left(\sigma_{Z}, \lambda_{Z}\right)=\sigma_{Z}^{2} \exp \left[-\frac{\left(v_{i}-v_{j}\right)^{2}}{2 \lambda_{Z}^{2}}\right]$

or the Matérn class of covariance functions,

$K_{i j}\left(\sigma_{Z}, \lambda_{Z}\right)=\sigma_{Z} \frac{2^{1-v}}{\Gamma(v)}\left(\frac{\sqrt{2 v}\left|v_{i}-v_{j}\right|}{\lambda_{Z}}\right)^{v} K_{v}\left(\frac{\sqrt{2 v}\left|v_{i}-v_{j}\right|}{\lambda_{Z}}\right)$,

where $K_{v}(x)$ is the modified Bessel function (see Abramowitz \& Stegun 1972) and $v$ is a real number controlling the roughness of the resulting functions. Matérn covariance functions converge to the squared exponential covariance function when $v \rightarrow \infty$. Other covariance functions can be found in Rasmussen \& Williams (2005). Figure 1 shows samples from a squared exponential GP prior with $\sigma_{Z}=1$ and different values of $\lambda_{Z}$. In the case of the squared exponential covariance function, one can see that the functions are smooth and the value of $\lambda_{Z}$ defines the rate of variability.

The two covariance functions described in the previous paragraph are stationary (Rasmussen \& Williams 2005), because they only depend on the difference $v_{i}-v_{j}$, so that they are translation invariant. The experiments carried out in Sects. 3 and 4 demonstrate that these covariance functions do a good job. Although we do not pursue this objective here, it is possible to work with nonstationary covariance functions that are more localized and could possibly explain some features in some specific velocity bins better (similar to the work of Czekala et al. 2015).

A GP prior introduces the parameters $\alpha_{Z}$ into the inference. These parameters of the prior are termed hyperparameters and have to be considered in the posterior distribution. This is the reason why the inference model is hierarchical. Therefore, the posterior distribution of the hierarchical model is now given by

$p\left(\boldsymbol{Z}, \boldsymbol{\alpha}_{Z} \mid \boldsymbol{V}\right)=\frac{p(\boldsymbol{V} \mid \boldsymbol{Z}) p\left(\boldsymbol{Z} \mid \boldsymbol{\alpha}_{Z}\right) p\left(\boldsymbol{\alpha}_{Z}\right)}{p(\boldsymbol{V})}$,

where we have added a hyperprior, $p\left(\alpha_{Z}\right)$, for the hyperparameters. Given that the hyperparameters are of no interest for obtaining the common signature, they should be marginalized (integrated) from the posterior so that we obtain the posterior distribution for $\boldsymbol{Z}$ :

$p(\boldsymbol{Z} \mid \boldsymbol{V})=\frac{1}{p(\boldsymbol{V})} \int \mathrm{d} \boldsymbol{\alpha}_{Z} p(\boldsymbol{V} \mid \boldsymbol{Z}) p\left(\boldsymbol{Z} \mid \boldsymbol{\alpha}_{Z}\right) p\left(\boldsymbol{\alpha}_{Z}\right)$

This integral cannot be computed in closed form in general. For this reason, we pursue here a Type-II maximum likelihood (ML) solution (Bishop 1996), also known as empirical Bayes or maximum marginal likelihood. This method is used for the analytical treatment of hierarchical models in which the posterior distribution is intractable. The strategy is to compute the marginal posterior for the hyperparameters,

$p\left(\boldsymbol{\alpha}_{Z} \mid \boldsymbol{V}\right)=\frac{1}{p(\boldsymbol{V})} p\left(\boldsymbol{\alpha}_{Z}\right) \int \mathrm{d} \boldsymbol{Z} p(\boldsymbol{V} \mid \boldsymbol{Z}) p\left(\boldsymbol{Z} \mid \boldsymbol{\alpha}_{Z}\right)$,

and assume that this distribution is strongly peaked. Under this condition the set of hyperparameters $\hat{\boldsymbol{\alpha}}_{Z}$ can be obtained that maximize the marginal posterior $p\left(\boldsymbol{\alpha}_{Z} \mid \boldsymbol{V}\right)$ and use a Dirac delta function as prior distribution:

$p\left(\boldsymbol{\alpha}_{Z}\right)=\delta\left(\boldsymbol{\alpha}_{Z}-\hat{\boldsymbol{\alpha}}_{Z}\right)$.

In such a case, the integral in Eq. (14) can be trivially carried out, so that we find

$p\left(\boldsymbol{Z} \mid \boldsymbol{V}, \hat{\boldsymbol{\alpha}}_{Z}\right)=\frac{1}{p(\boldsymbol{V})} p(\boldsymbol{V} \mid \boldsymbol{Z}) p\left(\boldsymbol{Z} \mid \hat{\boldsymbol{\alpha}}_{Z}\right)$,

where we make explicit in the posterior for $\boldsymbol{Z}$ that we are conditioning on a specific value of the hyperparameters.

The advantage of following this Type-II ML approach is that the solution becomes analytical. Making use of the definition of the likelihood of Eq. (6) and the prior of Eq. (10) with $\mu=0$ and applying standard properties of the multivariate Gaussian distribution, we find

$p\left(\boldsymbol{Z} \mid \hat{\boldsymbol{\alpha}}_{Z}, \boldsymbol{V}\right)=\mathcal{N}\left(\boldsymbol{Z} \mid \boldsymbol{\mu}_{Z}, \boldsymbol{\Sigma}_{Z}\right)$,

where

$\boldsymbol{\Sigma}_{Z}=\left[\mathbf{K}\left(\boldsymbol{\alpha}_{Z}\right)^{-1}+\mathbf{W}^{T} \operatorname{diag}\left(\frac{1}{\boldsymbol{\sigma}^{2}}\right) \mathbf{W}\right]^{-1}$
$\boldsymbol{\mu}_{Z}=\boldsymbol{\Sigma}_{Z} \mathbf{W}^{T} \operatorname{diag}\left(\frac{1}{\boldsymbol{\sigma}^{2}}\right) \boldsymbol{V}$.

Likewise, the marginal posterior for $\alpha_{Z}$ is

$p\left(\boldsymbol{\alpha}_{Z} \mid \boldsymbol{V}\right)=p\left(\boldsymbol{\alpha}_{Z}\right) \mathcal{N}\left(\boldsymbol{V} \mid \boldsymbol{\mu}_{\alpha}, \boldsymbol{\Sigma}_{\alpha}\right)$,

with

$\boldsymbol{\mu}_{\alpha}=0$

$\boldsymbol{\Sigma}_{\alpha}=\operatorname{diag}\left(\boldsymbol{\sigma}^{2}\right)+\mathbf{W K}\left(\boldsymbol{\alpha}_{Z}\right) \mathbf{W}^{T}$. 
The objective now is to obtain the value of $\hat{\boldsymbol{\alpha}}_{Z}$ that maximizes Eq. (20). This is easier if one takes logarithms, ending up with the maximization of

$\log p\left(\boldsymbol{\alpha}_{Z} \mid \boldsymbol{V}\right)=\log p\left(\boldsymbol{\alpha}_{Z}\right)-\frac{1}{2} \boldsymbol{V}^{T} \boldsymbol{\Sigma}_{\alpha}^{-1} \boldsymbol{V}-\frac{1}{2} \log \left|\boldsymbol{\Sigma}_{\alpha}\right|$,

where $\left|\Sigma_{\alpha}\right|$ is the determinant of the matrix.

From a technical point of view, the calculation of the inverse and the determinant is computationally heavy because matrix $\boldsymbol{\Sigma}_{\alpha}$ is of size $N_{\text {lines }} N_{v} \times N_{\text {lines }} N_{v}$, which is very large in practical applications. However, it is possible to efficiently accelerate the computation using the Woodbury matrix identity (Petersen \& Pedersen 2012), so that

$\boldsymbol{\Sigma}_{\alpha}^{-1}=\operatorname{diag}\left(\frac{1}{\sigma^{2}}\right)$

$-\operatorname{diag}\left(\frac{1}{\sigma^{2}}\right) \mathbf{W}\left[\mathbf{K}\left(\boldsymbol{\alpha}_{Z}\right)^{-1}+\mathbf{W}^{T} \operatorname{diag}\left(\frac{1}{\sigma^{2}}\right) \mathbf{W}\right]^{-1} \mathbf{W}^{T} \operatorname{diag}\left(\frac{1}{\sigma^{2}}\right)$.

In this case, it is only necessary to obtain the inverse of the $N_{v} \times$ $N_{v}$ matrix $\mathbf{K}\left(\boldsymbol{\alpha}_{Z}\right)$ and of the matrix inside brackets, which is again of size $N_{v} \times N_{v}$. These matrices are of a much smaller size than $\boldsymbol{\Sigma}_{\alpha}$ and the inversion is much less time consuming. Likewise, using the matrix determinant lemma, we find

$$
\begin{aligned}
\log \left|\boldsymbol{\Sigma}_{\alpha}\right|= & \log \left|\mathbf{K}\left(\boldsymbol{\alpha}_{Z}\right)^{-1}+\mathbf{W}^{T} \operatorname{diag}\left(\frac{1}{\boldsymbol{\sigma}^{2}}\right) \mathbf{W}\right| \\
& +\log \left|\mathbf{K}\left(\boldsymbol{\alpha}_{Z}\right)\right|+\log \left|\operatorname{diag}\left(\boldsymbol{\sigma}^{2}\right)\right| .
\end{aligned}
$$

The inverse of the matrix inside brackets can be efficiently computed using the Cholesky decomposition, which also gives the logarithm of the determinant as a subproduct.

As a final remark, we note that many of the terms in Eq. (23) do not change during the optimization with respect to the hyperparameters. They can be precomputed at the beginning of the optimization and only the term inside brackets in Eq. (23) needs to be recomputed when the hyperparameters are changed.

\section{Synthetic experiments}

The first test of the method is done with synthetic data. We generate two artificial $Z(v)$ profiles, which are given by

$$
\begin{aligned}
& Z_{1}(v)=\exp \left[-v^{2}\right] \\
& Z_{2}(v)=\exp \left[-v^{2}\right]+\frac{1}{5} \exp \left[-(v-2.5)^{2}\right] .
\end{aligned}
$$

For convenience, both profiles are normalized to $1 / 2$ amplitude, multiplied by randomly generated $\alpha$ coefficients (with zero mean and unit variance) and then noise with diagonal covariance with variance $\sigma^{2}=1$ is added to all the synthetic observations. A maximum of 200 synthetic spectral lines are used in both the standard LSD and the Bayesian LSD algorithms. For a single line, the signal-to-noise ratio equals $S / N=1 / 2$. In the presence of noise, it is expected that adding $N_{\text {lines }}$ spectral lines would increase the $\mathrm{S} / \mathrm{N}$ in proportion to $\sqrt{N_{\text {lines }}}$. Figure 2 shows how both estimations approach the true common signature (represented in red). The Bayesian LSD result is using a Matérn covariance function with $v=3 / 2$. The standard LSD method gives the green estimation. Given that no correlation is imposed between velocity bins, it can be seen that a large oscillation produced by noise remains even when adding 200 lines.
The mean of the marginal posterior for the common signature, given by $\boldsymbol{\mu}_{Z}$ in Eq. (19), is represented in blue. One of the obvious advantages of working in a Bayesian framework is that the solution is given in terms of a probability distribution. This allows us to compute the probability distribution for each velocity point. They are obtained by marginalizing out all the variables in Eq. (18) except for the value of $Z\left(v_{j}\right)$ at the velocity bin $j$ of interest. Using the special properties of the Gaussian distribution, these distributions are just one-dimensional Gaussian distributions with variance equal to the $(j, j)$ element of matrix $\boldsymbol{\Sigma}_{Z}$. We represent the distribution at each velocity point using the $68 \%$, $95 \%$, and $99 \%$ credibility intervals with blue shaded regions.

Several properties of the Bayesian LSD solution are worth mentioning. First, the mean of the solution is very smooth. This is a consequence of the strong regularization introduced by the GP prior with squared exponential covariance function. Other covariance functions that give a less smooth solution can also be used. Second, the covariance function is stationary (it only depends on $v-v^{\prime}$ ) so that we find some variability in the inferred solution when approaching the continuum because the scale of variation $\lambda_{Z}$ is the same for all the velocity bins. Many other covariance functions can be used to diminish this behavior. Third, the true solution is always inside the credibility intervals. Fourth, the marginal posterior of Eq. (14) can be used as a suitable distribution to carry out the modeling of the LSD profile. Using this distribution instead of the usual least squares solution (which assumes a diagonal covariance) makes the fitting much more robust.

\section{Real data}

The previous section demonstrates that Bayesian LSD can recover a much more robust common signature from the observations, even when it is quite complex. In the following, we apply the method to real observations. We consider three different cases that are representative of what one can encounter in typical observations. The case of II Peg is representative of a very strong signal that can even be detected in individual lines. The case of HR1099 is representative of a very complex common signature. Finally, $18 \mathrm{Sco}$ is representative of a very weak signal in which, even taking into account thousands of lines, the final signal is very close to the noise level. Public spectropolarimetric data for these three targets were downloaded from the PolarBase data base (Petit et al. 2014).

\subsection{Peg}

II Peg is a very active RS CVn binary system where a system of starspots has been mapped on the K1IV primary component. The spot system usually covers up to $40 \%$ of the surface (Berdyugina et al. 1998). The observations were carried out in July 2005 with the ESPaDOnS spectropolarimeter (Donati et al. 2006). Using a total integration time of one hour, the peak signal-tonoise ratio of the original Stokes $V$ spectrum (achieved at wavelengths around $780 \mathrm{~nm}$ ) is close to 1000 per wavelength bin. In agreement with the vast majority of published studies based on ESPaDOnS data, the $\mathrm{S} / \mathrm{N}$ used here is the one computed from the Stokes $I$ spectrum obtained by adding up the four polarimetric exposures combined to produce the Stokes $V$ spectrum. For such an extremely active target, this noise level is sufficiently low to detect Zeeman signatures in a number of individual spectral lines formed in the photosphere and chromosphere (Petit 2006). Here, we use a list of about 7400 spectral lines computed from a local thermodynamical equilibrium (LTE) atmospheric model 

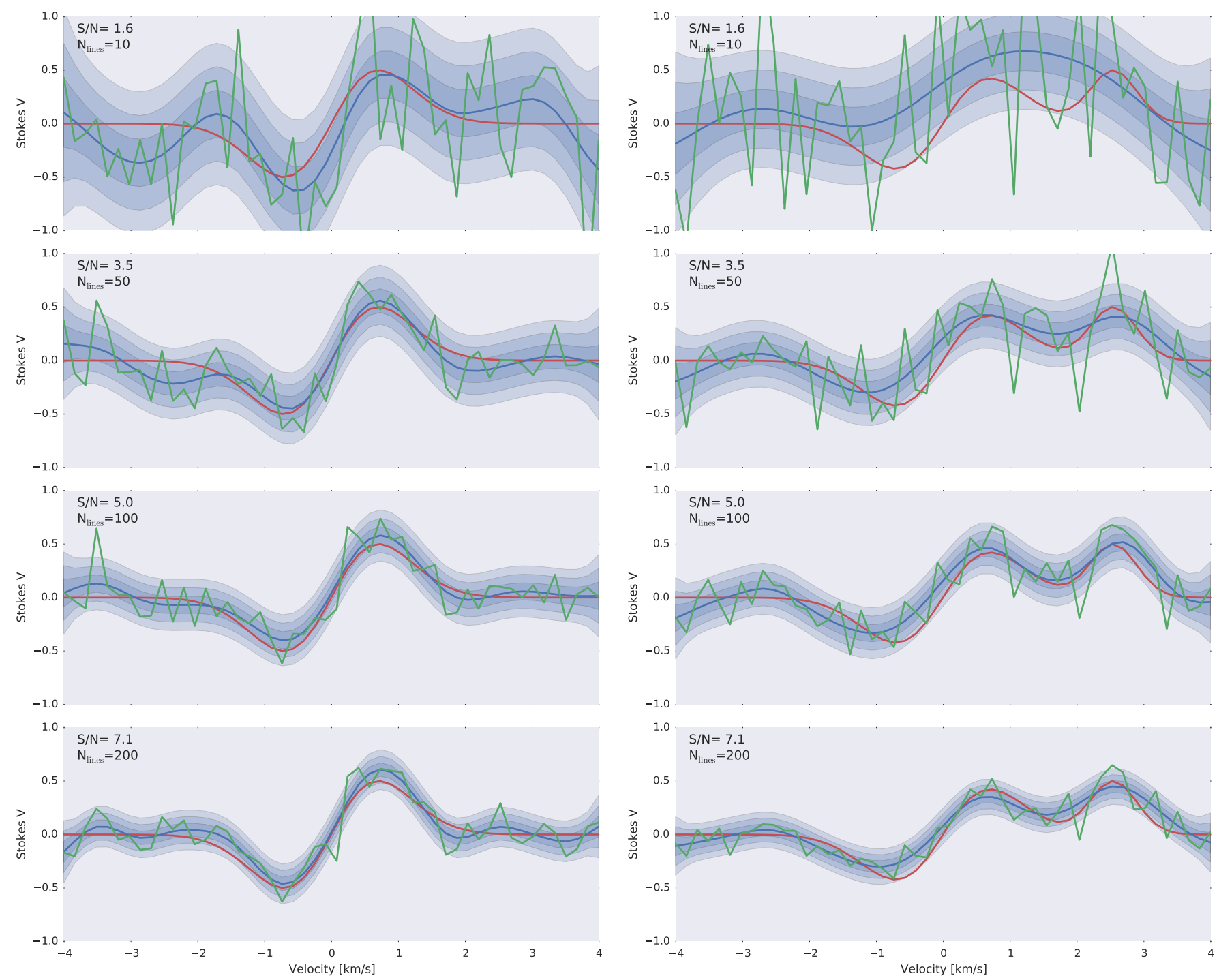

Fig. 2. Examples of two different shapes of $Z(v)$ and how they are recovered using LSD (green curve) and Bayesian LSD (mean shown in blue and credibility intervals as blue shaded regions). The original true $Z(v)$ is shown in red. The experiments use the Matern covariance function with $v=3 / 2$.

matching the spectral type of the primary component of II Peg (Kurucz 1993), keeping only photospheric lines deeper than $40 \%$ of the continuum level.

Figure 3 shows the improvement in the detection of the common signature $Z(v)$ when the number of lines that are considered increases. The left panel shows the common signature in Stokes $I$, while the right panel displays the results for Stokes $V$. The lines that we have considered have been ordered on decreasing value of the weights $\alpha_{i}$. Therefore, the panels with only seven lines are obtained by considering the lines that produce the largest Zeeman signal. The LSD profile is shown in green, while the mean of the Bayesian LSD detection is shown in blue, with the credibility intervals at $68 \%, 95 \%$, and $99 \%$ levels displayed in different gradations of blue. For the case of II Peg, the signature is detected at a very high signal-to-noise ratio with only seven lines $(0.1 \%$ of the total number of lines considered in the line list). The Bayesian LSD approach leads to a much smoother profile than the LSD does. The presence of credibility intervals is of relevance for discarding features like the one found at $v \approx-85 \mathrm{~km} \mathrm{~s}^{-1}$, which is compatible with zero in the Bayesian LSD case. Increasing the number of spectral lines produces a convergence of the Bayesian LSD and the standard LSD profiles, which is especially relevant in Stokes $V$.

\subsection{HR 1099}

Similarly to II Peg, HR 1099 is one of the most active RS CVn systems, with both components of the binary association clearly visible in high resolution spectra (while the primary alone is seen in observations of II Peg). The projected rotational velocity has been measured to be $v \sin i=41 \pm 0.5 \mathrm{~km} \mathrm{~s}^{-1}$ (Donati 1999). The magnetic structure of its active subgiant has been investigated in detail by Petit et al. (2004) using Zeeman Doppler Imaging, showing the presence of large and axisymmetric regions with the magnetic field being azimuthal. Additionally, Petit et al. (2004) also found clues for the presence of a differential rotation, much weaker than in the case of the Sun. The observation considered here was taken with ESPaDONS in August 2005.

The line list used here is the same as the one previously employed for II Peg, providing us with a good match to the photospheric characteristics of the evolved primary component, while the secondary is a much less active G5 main-sequence star. 

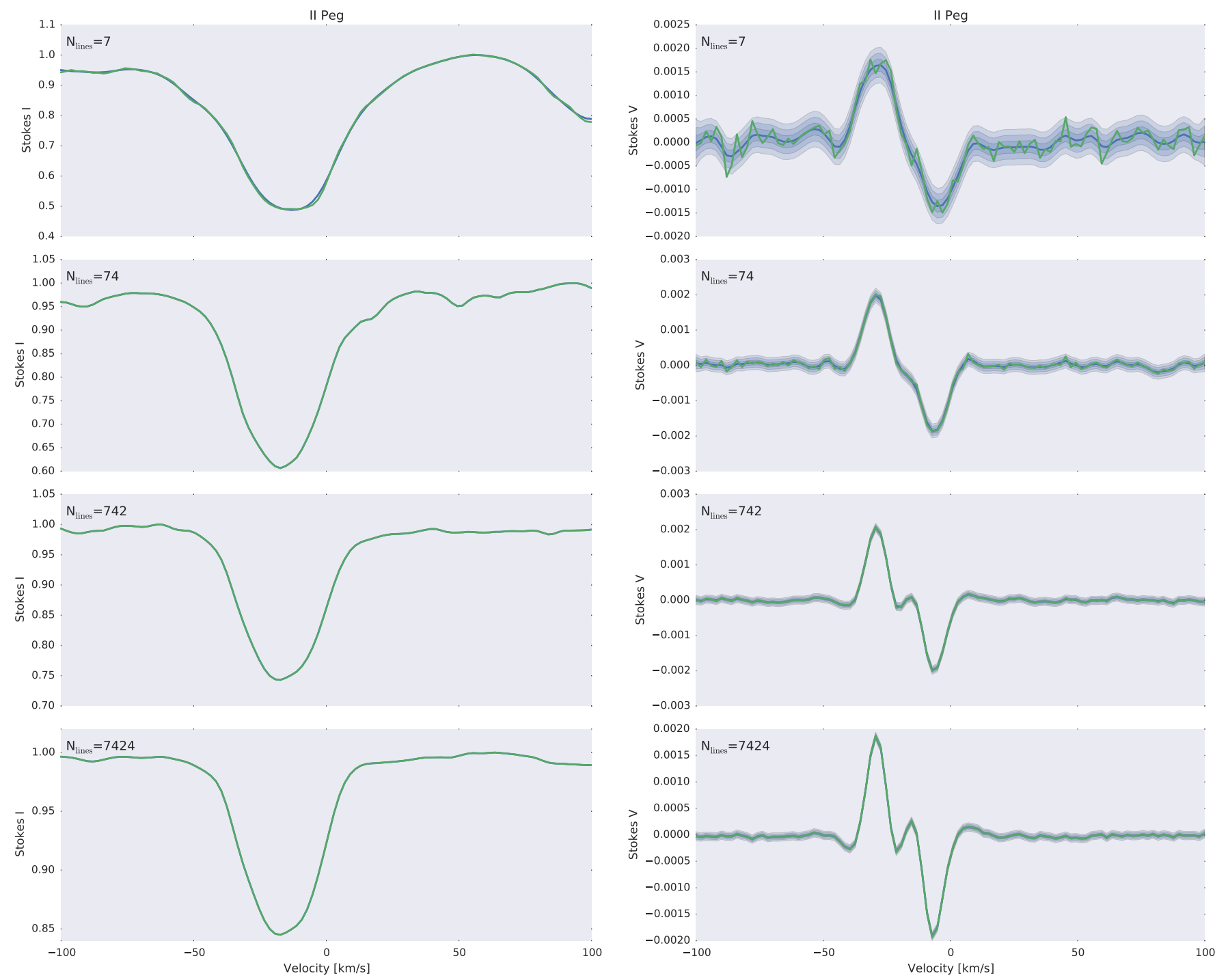

Fig. 3. Results of applying the LSD (green curve) and Bayesian LSD (blue curve showing the mean and blue regions showing the credibility intervals) to observations of II Peg, both for Stokes I (left panel) and V (right panel). The experiments use the Matérn covariance function with $v=3 / 2$. The green and blue curves overlap almost exactly in the left panel when increasing the number of considered spectral lines.

The presence of several magnetized regions that also change with time produces Zeeman signals of large complexity. Figure 4 shows the inferred common signature for HR 1099, both for Stokes $I$ (left panel) and for Stokes $V$ (right panel). Several magnetic components are detected around -65 (which is roughly the radial velocity of the secondary star at that time), 20 , and $40 \mathrm{~km} \mathrm{~s}^{-1}$ (originating from the surface of the primary star of the system). These detections in Stokes $V$ are also consistent with what is found in Stokes $I$. When only the seven most magnetically sensitive lines are considered, the mean of the Bayesian LSD profile for Stokes $V$ is very smooth with the dashed regions marking the large uncertainty. A large part of the noise that is intrinsic to the standard LSD profile is absent. With only 74 lines, the feature at positive relative velocities clearly appears. The quality of the detection increases drastically when considering more than $10 \%$ of the lines of the line list. However, it is interesting to note that the uncertainty in the continuum regions does not decrease much when using $10 \%$ or $100 \%$ of the lines (third and fourth panels). In the presence of only pure random errors with constant variance, this uncertainty should be reduced roughly in proportion to $\sqrt{N_{\text {lines }}}$. However, the assumption of constant variance in these observations is not correct because the $\mathrm{S} / \mathrm{N}$ changes greatly throughout the ESPaDOnS spectrum, being much lower at the borders of individual spectral orders and also near the boundaries of the spectral domain of the instrument. This leads to a slower decrease in the final uncertainty. It is important to point out that our Bayesian LSD method is able to take this into account and the ensuing final uncertainties do not decrease in such a proportion. The immediate consequence of this is that we could be missing very weak signals owing to the presence of systematic errors.

\section{3. $18 \mathrm{Sco}$}

18 Sco (HD 146233) is known to be one of the best solar twins among bright stars (Meléndez \& Ramírez 2007). In spite of its relatively low activity level, its surface magnetic field was detected by Petit et al. (2008) using high S/N observations. The Stokes $V$ spectrum analyzed here was obtained with the NARVAL spectropolarimeter (Aurière 2003) in July 2008. This observation is extracted from the time-series presented by Petit 


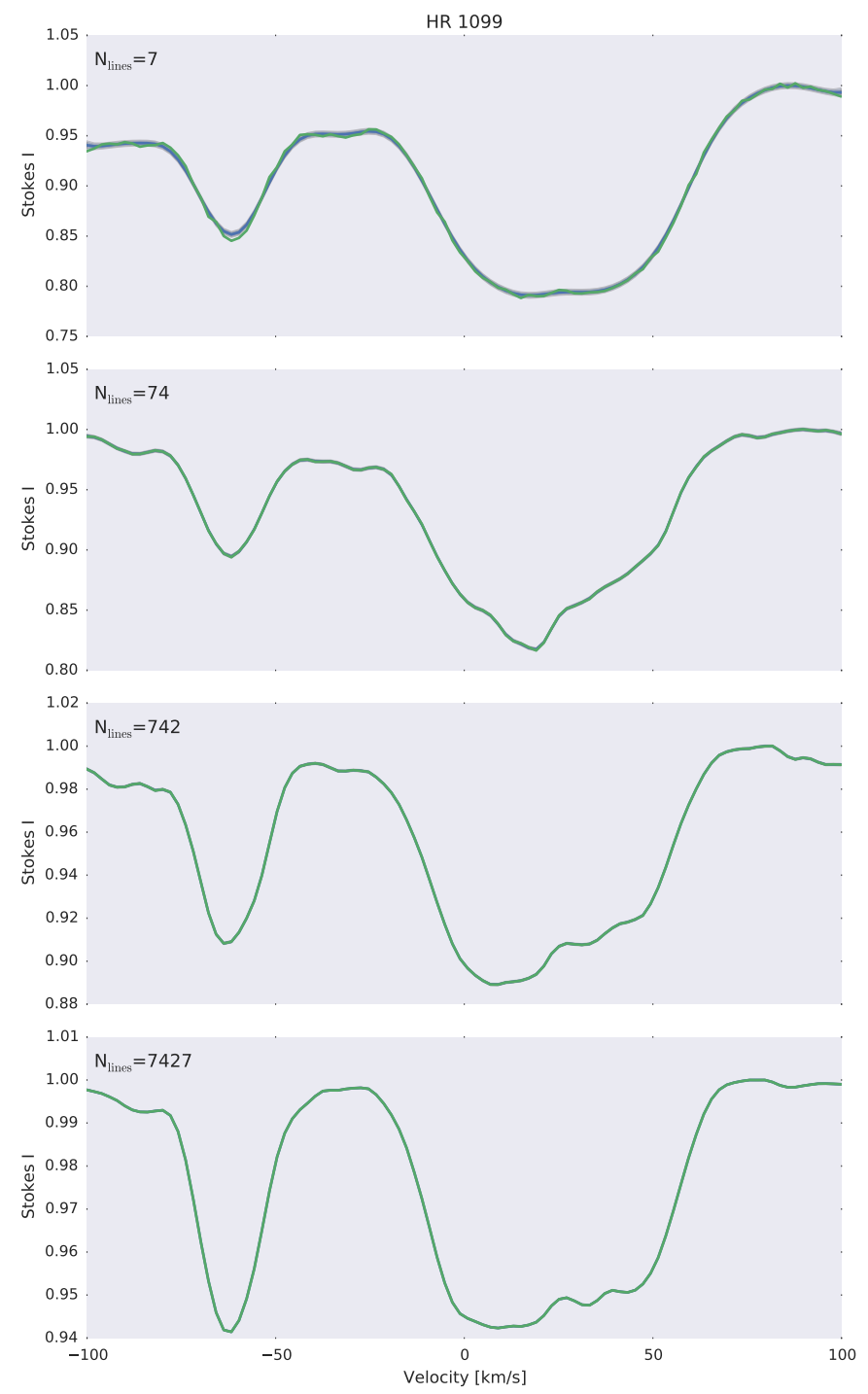

Fig. 4. Same as Fig. 3 for HR1099.

et al. (2008). Our line list is also identical to the one used in the same study. The LTE model from Kurucz (1993) was computed for a G2 spectral type, leading to a total of about 3700 photospheric spectral lines deeper than $40 \%$ of the continuum.

The inferred common signature for 18 Sco is shown in Fig. 5 for an increasing number of spectral lines. Even when the full line list is considered, only a marginal detection of circular polarization is possible at the radial velocity of the star. This is an example in which having credibility intervals for each velocity bin is crucial. The oscillations detected by the standard LSD method fall inside the uncertainty region obtained from the Bayesian LSD method, although in this case the mean is much smoother.

\section{Conclusions}

We have described a fully Bayesian least squares deconvolution method which relies on a Gaussian process prior to regularizing the spectral shape of the common signature. Two obvious advantages emerge from this Bayesian approach. First, the final answer to the problem is in the form of a probability distribution. This allows the user to check whether the detected signals are relevant or just a consequence of fluctuations of random and/or
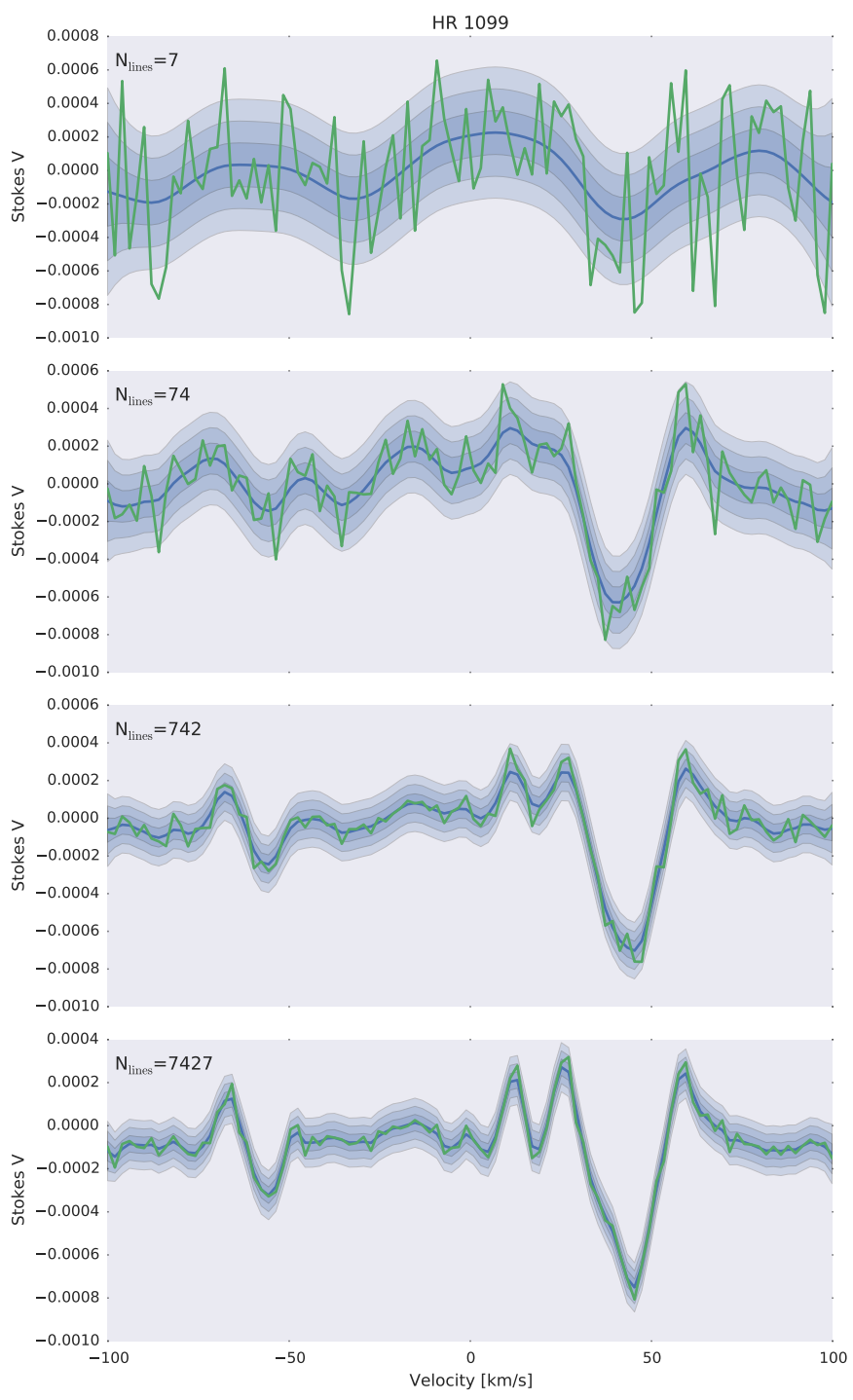

systematic effects. Additionally, given that we also compute the covariance matrix of the ensuing probability distribution (taking into account possible correlations among all velocity bins), it can be used in inversion codes that fit the Bayesian LSD profile to estimate the stellar magnetic field. This introduces a strong regularization that avoids fitting spurious effects. Examples of this are features of the LSD profiles that are close to the noise level or systematic effects like interference fringes. Second, the hierarchical approach using a GP prior automatically adapts to the complexity of the spectral profile. The solution for very noisy velocity bins (for instance, close to the continuum) is very smooth and the noise is never overfitted. This automatic adaptation to the signal is allowed owing to the inclusion of the two hyperparameters of the GP prior. They are easily inferred from the data.

By taking advantage of standard linear algebra identities and techniques for working with sparse matrices, the Bayesian LSD common signature can be computed very quickly, in a matter of a few seconds for thousands of spectral lines. The code for computing the Bayesian LSD profile is freely available ${ }^{1}$.

1 https://github.com/aasensio/pyGPLSD 

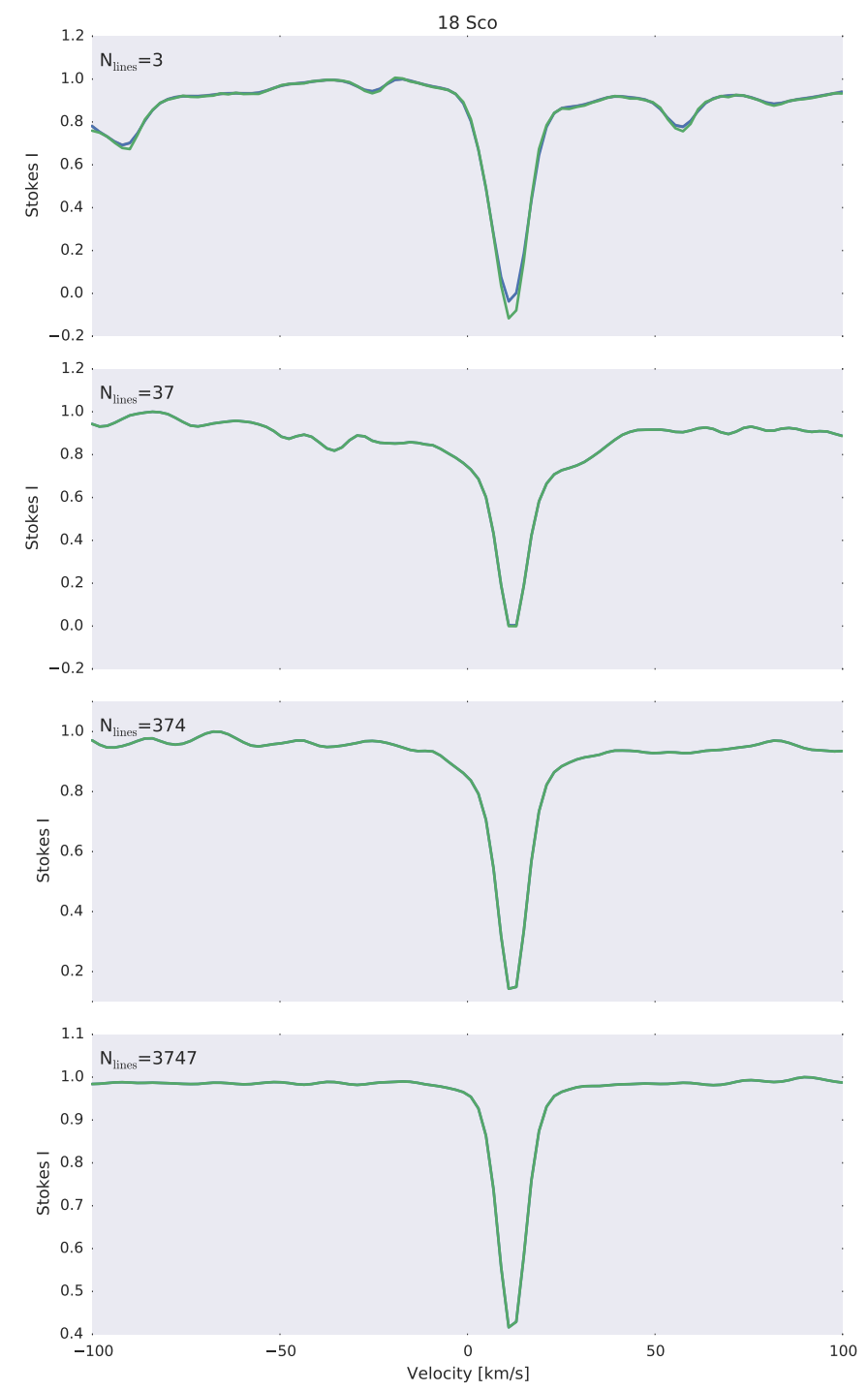

Fig. 5. Same as Fig. 3 for 18 Sco.

Acknowledgements. Financial support by the Spanish Ministry of Economy and Competitiveness through projects AYA2010-18029 (Solar Magnetism and Astrophysical Spectropolarimetry) and Consolider-Ingenio 2010 CSD200900038 are gratefully acknowledged. A.A.R. also acknowledges financial support through the Ramón y Cajal fellowships. This research has made use of NASA's Astrophysics Data System Bibliographic Services.

\section{References}

Abramowitz, M., \& Stegun, I. A. 1972, Handbook of Mathematical Functions (New York: Dover)

Aurière, M. 2003, in EAS Pub. Ser. 9, eds. J. Arnaud, \& N. Meunier, 105

Berdyugina, S. V., Jankov, S., Ilyin, I., Tuominen, I., \& Fekel, F. C. 1998, A\&A, 334,863

Bishop, C. M. 1996, Neural networks for pattern recognition (Oxford University Press)

Carroll, T. A., Kopf, M., Ilyn, I., \& Strassmeier, K. G. 2008, Astron. Nachr., 328, 1043

Czekala, I., Andrews, S. M., Mandel, K. S., Hogg, D. W., \& Green, G. M. 2015, ApJ, 812, 128

Donati, J.-F. 1999, MNRAS, 302, 457

Donati, J.-F., Semel, M., \& Rees, D. E. 1992, A\&A, 265, 669

Donati, J.-F., Semel, M., Carter, B. D., Rees, D. E., \& Collier Cameron, A. 1997, MNRAS, 291, 658

Donati, J.-F., Catala, C., Landstreet, J. D., \& Petit, P. 2006, in ASP Conf. Ser. 358, eds. R. Casini, \& B. W. Lites, 362
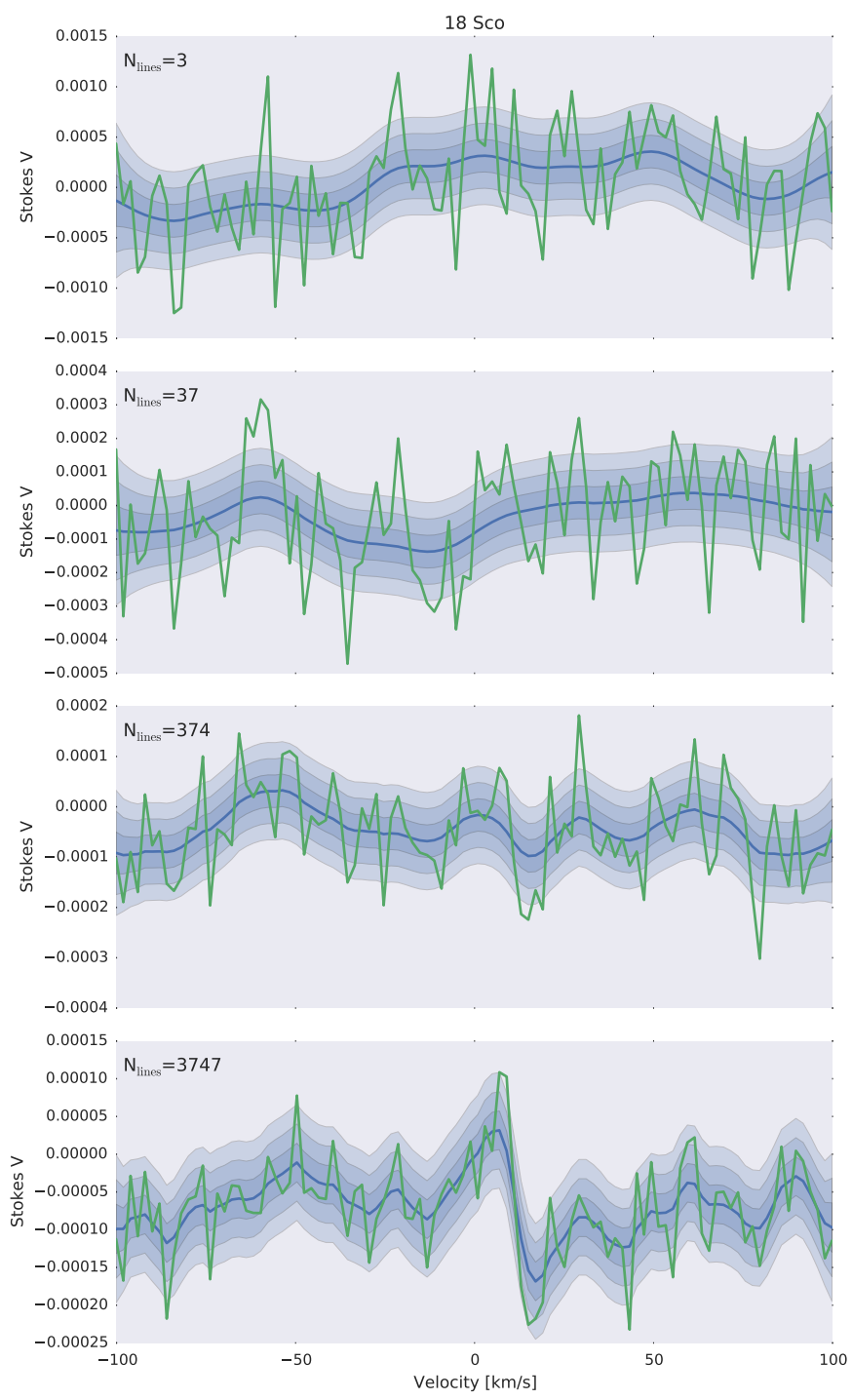

Kochukhov, O., Makaganiuk, V., \& Piskunov, N. 2010, A\&A, 524, A5

Kurucz, R. L. 1993, SYNTHE spectrum synthesis programs and line data (Cambridge, MA: Smithsonian Astrophysical Observatory)

Landi Degl'Innocenti, E., \& Landolfi, M. 2004, Polarization in Spectral Lines (Kluwer Academic Publishers)

Marsden, S. C., Petit, P., Jeffers, S. V., et al. 2014, MNRAS, 444, 3517

Martínez González, M. J., Asensio Ramos, A., Carroll, T. A., et al. 2008, A\&A, 486, 637

Martins, F., Hervé, A., Bouret, J.-C., et al. 2015, A\&A, 575, A34

Meléndez, J., \& Ramírez, I. 2007, ApJ, 669, L89

Petersen, K. B., \& Pedersen, M. S. 2012, The Matrix Cookbook, version 20121115

Petit, P. 2006, in ASP Conf. Ser. 358, eds. R. Casini, \& B. W. Lites, 335

Petit, P., Donati, J.-F., Wade, G. A., et al. 2004, MNRAS, 348, 1175

Petit, P., Dintrans, B., Solanki, S. K., et al. 2008, MNRAS, 388, 80

Petit, P., Louge, T., Théado, S., et al. 2014, PASP, 126, 469

Rasmussen, C. E., \& Williams, C. K. I. 2005, Gaussian Processes for Machine Learning, Adaptive Computation and Machine Learning (The MIT Press)

Semel, M. 1989, A\&A, 225, 456

Semel, M., \& Li, J. 1996, Sol. Phys., 164, 417

Semel, M., Ramírez Vélez, J. C., Martínez González, M. J., et al. 2009, A\&A, 504,1003

Sennhauser, C., Berdyugina, S. V., \& Fluri, D. M. 2009, A\&A, 507, 1711

Wade, G. A., Grunhut, J. H., \& MiMeS Collaboration 2012, in Circumstellar Dynamics at High Resolution, eds. A. C. Carciofi, \& T. Rivinius, ASP Conf. Ser., 464, 405 